\title{
Glucose enhancement of tolerance to morphine and ethanol in rats
}

\author{
SHEPARD SIEGEL \\ McMaster University, Hamilton, Ontario, Canada
}

\begin{abstract}
According to a Pavlovian conditioning analysis, drug tolerance (in part) results from an association between cues present at the time of drug administration and the effect of the drug. Associations are enhanced by glucose administered after a conditioning trial, such enhancement being inversely related to the interval between the trial and glucose administration. If conditioning contributes to tolerance, glucose administered shortly after each drug administration should facilitate the acquisition of tolerance. Acquisition of tolerance was assessed both when glucose was administered shortly after each drug administration (immediate glucose) and when glucose was administered $24 \mathrm{~h}$ after each drug administration. As expected on the basis of the conditioning interpretation of tolerance, immediate glucose facilitated the acquisition of tolerance to the analgesic effect of morphine (Experiment 1 ) and the ataxic effect of ethanol (Experiment 2).
\end{abstract}

Tolerance, a decrease in responsivity to a drug over the course of repeated administrations, can be affected by drug-associated cues. The contribution of drug-associated cues to tolerance is emphasized in a Pavlovian conditioning analysis of tolerance (see reviews by Dworkin, 1993; Ramsay \& Woods, 1997; Siegel \& Allan, 1998). Using the usual conditioning terminology, cues accompanying the primary drug effect function as conditioned stimuli (CSs). The direct effect of the drug constitutes the unconditioned stimulus (UCS). Prior to any learning, this pharmacological stimulation elicits responses that compensate for the drug-induced disturbances. These responses that compensate for the drug effect are unconditioned responses (UCRs). After some pairings of the predrug CS and pharmacological UCS, a drug-compensatory response is elicited as a conditioned response (CR). These drugcompensatory CRs mediate the development of tolerance by counteracting the drug effect.

Consistent with the conditioning analysis, a variety of manipulations that attenuate the expression of conditional responding should also attenuate the acquisition of tolerance. Thus, in common with other CRs, the expression of drug tolerance is disrupted when a novel external stimulus is presented (external inhibition, see, e.g., Larson \& Siegel, 1998; Siegel \& Larson, 1996) or when the putative $\mathrm{CS}$ is altered (the context of drug administration changed, e.g., Epstein, Caggiula, Perkins, McKenzie, \& Smith, 1991; Siegel, 1991). Even if the drug is reliably signaled by consistent environmental cues, the expression of tol-

This research was supported by grants from the Alcoholic Beverage Medical Research Foundation and the Natural Sciences and Engineering Research Council of Canada. The invaluable assistance of Doreen Mitchell is gratefully acknowledged. Correspondence should be addressed to S. Siegel, Department of Psychology, McMaster University, Hamilton, ON, L8S 4K1 Canada (e-mail: siegel@mcmaster.ca). erance is attenuated by compound conditioning phenomena, such as overshadowing (see, e.g., Walter \& Riccio, 1983) and blocking (see, e.g., Dafters, Hetherington, \& McCartney, 1983). The acquisition of tolerance is retarded by partial reinforcement (see, e.g., Krank, Hinson, \& Siegel, 1984), CS preexposure (so-called latent inhibition, see, e.g., Tiffany \& Baker, 1981), and inhibitory learning (see, e.g., Hinson \& Siegel, 1986). Posttrial events, such as electroconvulsive shock or frontal cortical stimulation, which affect memory consolidation and decrease the strength of learning, similarly decrease the acquisition of morphine tolerance (see Siegel, 1983).

On the basis of the conditioning analysis of tolerance, it would also be expected that manipulations that enhance conditioning should similarly enhance the acquisition of tolerance. In recent years there has been increasing evidence that simple glycemic manipulations, applied immediately after a CS-UCS pairing, facilitate learning. Injection of glucose after a trial facilitates learning in mice and rats, and oral consumption of glucose facilitates learning in humans. Moreover, lowering blood glucose after a trial (by insulin injection) decreases the strength of the association formed on that trial. The posttraining treatments presumably modulate memory storage processes because the effects of glucose and insulin on memory are time dependent. That is, the effects are maximal if the glycemic manipulations occur immediately after a trial and are minimal to nonexistent if the manipulations are delayed, for example, for a few hours (for reviews of glycemic manipulations and conditioning, see Manning, Parsons, Cotter, \& Gold, 1997; Okaichi \& Okaichi, 1997; Winocour, 1995, 1998).

If drug tolerance is, in part, a manifestation of an association between drug administration cues and the systemic effect of the drug, the formation of this association, and hence the development of tolerance, should be enhanced in subjects receiving glucose shortly after each drug ad- 
ministration. In these experiments, we evaluated the effects of glucose on morphine and ethanol tolerance in rats.

\section{EXPERIMENT 1}

Experiment 1 was designed to evaluate the effect of glucose on the acquisition of tolerance to the analgesic effect of morphine. Rats assigned to morphine groups were injected with the opiate in a distinctive environment on alternate days, and analgesia was assessed following each morphine injection. The dose of morphine used, $5 \mathrm{mg} / \mathrm{kg}$, was the same as that used in many prior experiments, from our laboratory and elsewhere (see Siegel, 1991), to evaluate the contribution of conditioning to morphine tolerance. Approximately half the morphineinjected rats were injected with glucose immediately following each analgesia assessment (mor-immediate group). These mor-immediate rats were injected with physiological saline on those alternate days when they did not receive morphine. The remaining morphine-injected rats were not injected with glucose following each morphine administration; rather, they were injected with physiological saline (mor-delayed group). These mor-delayed rats were injected with glucose on those alternate days when they did not receive morphine; thus the interval between morphine and glucose was $24 \mathrm{~h}$ for delayed-group rats. The dose of glucose used, $120 \mathrm{mg} / \mathrm{kg}$, is similar to that used by others who have reported glucose facilitation of learning (e.g., Winocour, 1995). In addition, two further groups of rats were injected with saline, rather than morphine, on each tolerance development session. Thus, rats in Groups Sal-Immediate and Sal-Delayed were treated the same as rats in Groups Mor-Immediate and MorDelayed, except that analgesia was assessed following injections of saline, rather than the opiate.

\section{Method}

Subjects and Apparatus. The subjects were 55 male LongEvans derived rats, 7-8 months old at the beginning of the experiment. None had had any exposure to morphine (or any other drug) or analgesia assessment prior to the start of the experiment, but some had been injected with physiological saline in the past (having served in control groups in other experiments). An attempt was made to equate the groups with respect to subjects' preexperimental history.

Analgesia was measured with the tail-flick procedure (Fennessy $\&$ Lee, 1975). The tail of a lightly restrained rat was immersed $5 \mathrm{~cm}$ into a water bath maintained at $50^{\circ} \mathrm{C}$. The latency for the rat to lift its tail out of the water was noted.

All injections were intraperitoneal (i.p.). Morphine sulfate, prepared as a $5-\mathrm{mg} / \mathrm{ml}$ solution, was injected at a dose of $5 \mathrm{mg} / \mathrm{kg}$. Glucose, prepared as a $120-\mathrm{mg} / 10 \mathrm{ml}$ solution, was injected at a dose of $120 \mathrm{mg} / \mathrm{kg}$. Saline injections were equated volumetrically with glucose injections.

Procedure. Once every other day all subjects were taken, in their home cages, from the colony room to the distinctive room containing the water bath. Fifteen minutes after transport, they were injected with morphine (Groups Mor-Immediate and Mor-Delayed, $n=18$ and 17 per group, respectively) or physiological saline (Groups Sal-Immediate and Sal-Delayed, $n$ per group $=10$ ). They remained in the distinctive room for $30 \mathrm{~min}$, and analgesia was as- sessed. Immediately following analgesia assessment, immediategroup rats were injected with glucose, and delayed-group rats were injected with saline. Twenty-four hours later, immediate-group rats were injected with saline (in the colony room), and delayed-group rats were injected with glucose. Each rat received five bidaily sessions (and analgesia assessments) over 10 days.

\section{Results and Discussion}

The mean tail-flick latencies $( \pm 1 S E M)$ for all groups across the five sessions are shown in Figure 1. The analgesic effect of morphine is clear in Figure 1; morphineinjected rats displayed longer response latencies than did saline-injected rats. Morphine-induced analgesia decreased over the course of the five sessions; that is, tolerance developed. This tolerance was more pronounced in mor-immediate rats than in mor-delayed rats. Glucose had no effect on response latencies of saline-injected rats (i.e., the latencies of sal-immediate rats and sal-delayed rats were similar).

A mixed-design analysis of variance (ANOVA) of the data followed by planned comparisons (LSD) confirmed the trends apparent in Figure 1. The groups $\times$ sessions interaction was significant $[F(12,204)=10.5, p<.001]$. On each of the five sessions, rats assigned to morphine groups responded more slowly to the thermal stimulation than rats assigned to the saline groups (all $p \mathrm{~s}<.001$ ). Rats assigned to the mor-immediate group acquired tolerance more rapidly than rats assigned to the mor-delayed group. Although the two groups did not significantly differ on the first two sessions, response latencies were shorter in mor-immediate rats than in mor-delayed rats

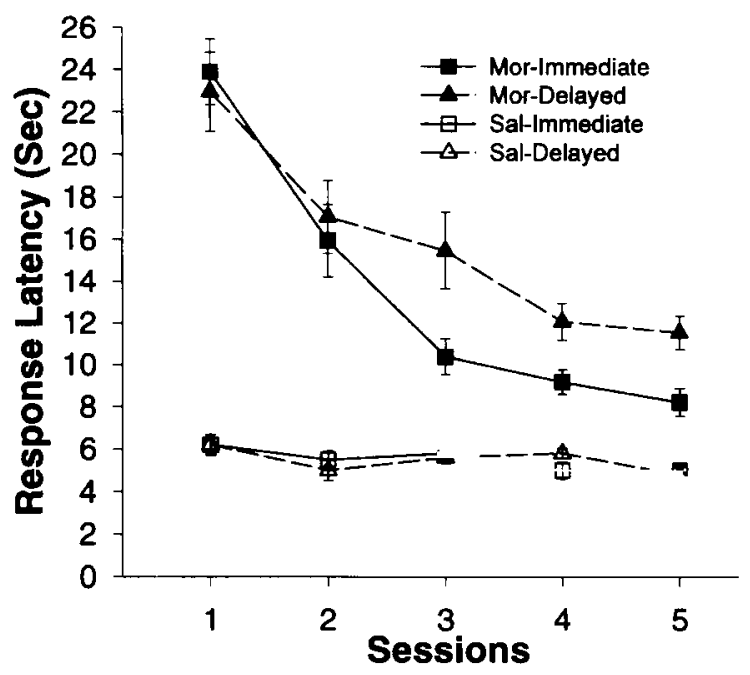

Figure 1. Mean ( $\pm 1 S E M$ ) response latency (tail-flick from $50^{\circ} \mathrm{C}$ water) for groups in Experiment 1 . Rats in two groups were administered morphine on each session and injected with glucose either immediately after analgesia assessment (mor-immediate) or $24 \mathrm{~h}$ after analgesia assessment (mor-delayed). Rats in the remaining groups were administered saline on each session, followed by glucose either immediately after analgesia assessment or 24 h later (Groups Sal-Immediate and Sal-Delayed, respectively). 
on each of the remaining three sessions (all $p s<.01$ ). None of the differences between the two saline groups approached statistical significance.

As expected on the basis of a conditioning analysis of tolerance, glucose facilitated the acquisition of tolerance to the analgesic effect of morphine. The effect of glucose on tolerance, like the effect of glucose on other CRs, is time dependent. Glucose administered shortly after the administration of the morphine (mor-immediate) enhanced tolerance more than glucose administered $24 \mathrm{~h}$ after morphine (mor-delayed).

\section{EXPERIMENT 2}

Experiment 2 was designed to evaluate the effect of glucose on the acquisition of tolerance to the ataxic effect of ethanol. The design of Experiment 2 was similar to that of Experiment 1 except there were no saline groups. All rats were injected with ethanol in a distinctive environment on alternate days, and ataxia was assessed following each ethanol injection. Approximately half the rats were injected with glucose immediately following each ataxia assessment (immediate group) and were injected with saline $24 \mathrm{~h}$ later. The remaining rats (delayed group) were injected with saline following each assessment of ethanol-induced ataxia and were injected with glucose $24 \mathrm{~h}$ later.

\section{Method}

Subjects. The subjects were 29 female and 4 male Long-Evans derived rats, 7-8 months old at the beginning of the experiment. Seventeen subjects ( 15 female and 2 male) were assigned to the immediate group, and 16 (14 female and 2 male) to the delayed group. None had had any exposure to ethanol (or any other drug) or ataxia assessment procedure prior to the start of the experiment, but some had been injected with physiological saline in the past (having served in control groups in other experiments). Some subjects were retired breeders. An attempt was made to equate the two groups with respect to subjects' preexperimental history.

Apparatus. The ataxic effect of ethanol was assessed with a tilting plane (Arvola, Sammalisto, \& Wallgren, 1958; Siegel \& Larson, 1996). The apparatus consisted of an alley, $60 \mathrm{~cm}$ long $X$ $18 \mathrm{~cm}$ wide, constructed of Plexiglas. It was enclosed by walls $30 \mathrm{~cm}$ high and open at the top. The alley was hinged at one end. The other end could be elevated by the operation of a crank (one complete revolution of the crank elevated the apparatus approximately $2^{\circ}$ ). A protractor built into the hinged end of the apparatus provided an indication of the angle of inclination. The ataxic effect of ethanol was measured by an experimenter who gradually turned the crank and noted the angle of inclination at which the rat started slipping down the alley (slip angle).

All injections were i.p. Ethanol, injected at a dose of $1.5 \mathrm{~g} / \mathrm{kg}$, was prepared as a $15 \%$ solution (by volume) of $95 \%$ ethanol in physiological saline. This dose of ethanol is similar to that used in other studies of the associative basis of ethanol tolerance, both in this laboratory (e.g., Larson \& Siegel, 1998) and elsewhere (e.g., Mansfield \& Cunningham, 1980). As in Experiment 1, the glucose dose was $120 \mathrm{mg} / \mathrm{kg}$.

Procedure. All subjects were injected with ethanol at 48-h intervals. For each session, subjects were taken from their home cage in the colony room to the room containing the tilt apparatus. Within 2 min of transport, they received a preinjection slip-angle assessment. They then were injected with ethanol, and slip angle was again determined at 2-min intervals for $14 \mathrm{~min}$. Subjects assigned to the immediate group were injected with glucose immediately after the final daily slip-angle assessment. Subjects assigned to the delayed group were injected with saline after this assessment. Subjects were returned to their home cages following the postethanol injection. Twenty-four hours later, immediate-group rats were injected with saline (in the colony room), and delayed-group rats were injected with glucose. Each rat received six ethanol injections (and ataxia assessments) over 12 days.

\section{Data Treatment}

For each subject, for each session, an impairment score was computed. The score is the difference, in degrees, between the mean of the seven postinjection slip-angle assessments and the preinjection assessment. Thus, increasing ataxia is indexed by increasingly negative impairment scores.

\section{Results and Discussion}

The variables of subject preexperimental history (saline injection, breeder) and subject sex did not significantly affect the results, which are presented collapsed across these variables.

The mean $( \pm 1 S E M)$ impairment scores for immediateand delayed-group rats for each ethanol session are shown in Figure 2. As would be expected, rats in the two groups displayed similar levels of impairment on the first session, prior to differential treatment. The effects of the group treatments were apparent by the second session. Immediate-group rats (injected with glucose about $14 \mathrm{~min}$ following the first ethanol injection) displayed less impairment on the second session than did delayed-group rats (injected with glucose about $24 \mathrm{~h}$ following the first ethanol injection). The differences between the groups persisted throughout the remaining sessions; immediategroup rats displayed increasingly less ethanol-induced

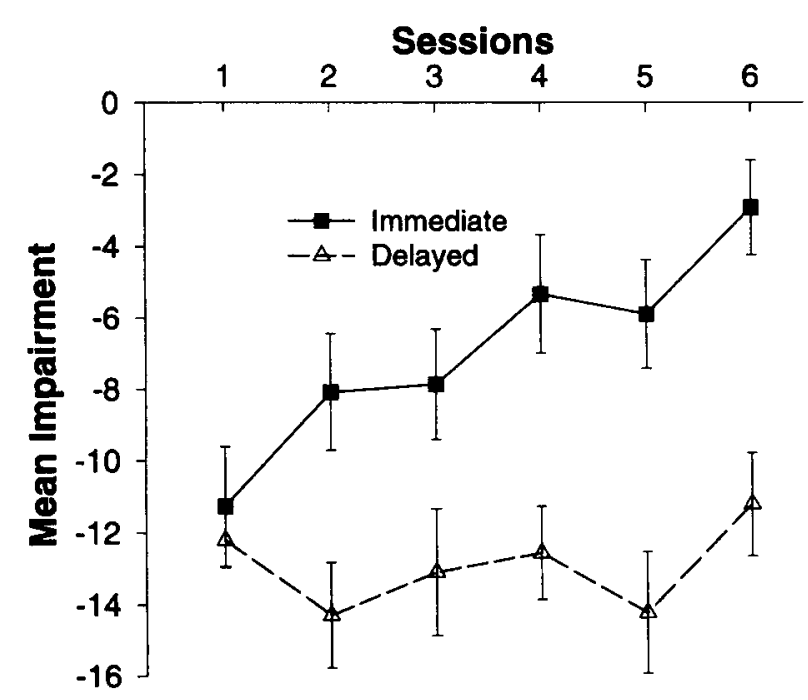

Figure 2. Mean ( $\pm 1 S E M$ ) impairment scores for groups in Experiment 2. Greater ataxia is denoted by increasingly negative impairment scores. Rats in both groups were administered ethanol on each session and injected with glucose immediately either after measurement of ataxia (immediate) or $24 \mathrm{~h}$ later (delayed). 
ataxia, but delayed-group rats showed no evidence of such tolerance.

An ANOVA followed by planned comparisons (LSD) confirmed the trends apparent in Figure 2. Although immediate- and delayed- group rats did not differ significantly on the first session $(p>.6)$, they did on every subsequent session (all $p \mathrm{~s}<.01$ ). Rats assigned to the immediate group displayed a significant decrease in ethanolinduced ataxia across sessions $[F(5,80)=3.8, p<.004]$, but no such trend was apparent for rats assigned to the delayed group $[F(5,75)<1]$.

The present finding that glucose facilitates the acquisition of tolerance to the ataxic effect of ethanol provides further support for the conditioning analysis of such tolerance. In this experiment, delayed-group rats, in contrast to immediate-group rats, displayed no evidence of tolerance. In other experiments (not involving the administration of glucose), we have reported that ataxic tolerance is seen with this preparation (Larson \& Siegel, 1998; Siegel \& Larson, 1996). It is possible that one reason for the difference is the age of the rats. In the earlier studies, we used rats younger than those used in the present experiment (approximately 3 months old vs. 7-8 months old). We used the older rats in the present experiment because there is evidence that ethanol tolerance is slower in older rats than in younger rats (see, e.g., Mayfield, Grant, Schallert, \& Spirduso, 1992; York \& Chan, 1994), thus increasing the possibility that glucose-induced enhancement of tolerance would be apparent.

\section{GENERAL DISCUSSION}

In each experiment, two groups of drug-injected rats received the same doses of the drug (morphine and ethanol, in Experiments 1 and 2, respectively), at the same intervals, the same number of times. Also, in each experiment both groups of drug-injected rats received the same dose of glucose at the same intervals the same number of times (thus, any differences between groups cannot be attributed merely to repeated drug and glucose administrations). Moreover, in each experiment, the glucose was administered after each drug administration and assessment of the drug effect; thus differences between groups cannot be attributed to differences in direct glucose modulation of the drug effect. In both experiments, the druginjected group that received the glucose shortly after drug administration displayed faster tolerance acquisition than the group that received the glucose $24 \mathrm{~h}$ after drug administration. These demonstrations that glucose facilitates the acquisition of tolerance are consistent with the conditioning analysis of tolerance. That is, the acquisition of tolerance, in common with the acquisition of other classically conditioned CRs, is enhanced by posttrial glucose.

Although the results of the present experiments are consistent with a Pavlovian conditioning analysis of tolerance, there are other ways in which learning may contribute to tolerance (see, e.g., Cunningham, Losli, \& Risinger, 1992). For example, Dews (1962) suggested that the frequently drugged subject may acquire a behavioral strategy that compensates for some drug-induced impairments. Dews's example clearly illustrates the operation of such an instrumentally acquired ability to cope with the effects of ethanol: The experienced drinker is more proficient in remaining erect than the inexperienced drinker because the experienced drinker has instrumentally acquired a behavioral strategy (a broad-based gait) that compensates for the effect of ethanol because he/she has practiced this behavior while intoxicated. In Experiment 2, rats assessed on the tilting plane after ethanol administration may have learned to make postural adjustments that caused them to resist slipping as the assessment apparatus was tilted. Similarly, it is possible that in Experiment 1, rats tested for tail-flick latency after morphine administration may have learned to make the analgesia-indicant response while narcotized (although there was no evidence of such learning in saline-injected rats). The relationship between instrumental conditioning and classical conditioning accounts of tolerance has been a matter of considerable discussion (e.g., Goodison \& Siegel, 1995; Hayes \& Mayer, 1978; Poulos \& Cappell, 1991; Siegel, 1978). However, instrumental conditioning is enhanced by posttrial glucose (see, e.g., Winocour, 1995); thus, demonstrations that glucose facilitates tolerance are consistent with an instrumental conditioning analysis of such tolerance, as well as a Pavlovian conditioning analysis.

The mechanisms by which glucose facilitates learning are not well understood, but it appears that glucose facilitates release or synthesis of acetylcholine, and this enhancement of central cholinergic function is responsible for the effect of glucose (see, e.g., Kopf \& Baratti, 1994). Indeed, various anticholinesterase inhibititors, in common with glucose, facilitate learning (see, e.g., Alvarez et al., 1997).

Cholinergic function declines with age (see, e.g., Tariot, Patel, Cox, \& Henderson, 1996), and, as discussed previously, rats that are more aged than those typically used in behavioral research were used as subjects in these experiments to increase the likelihood of detecting glucose enhancement of tolerance. The fact that the rate of development of tolerance to a variety of drugs is slower in older than in younger subjects (see, e.g., Mayfield et al., 1992; Nicak \& Kohut, 1978; Nozaki, Akera, Lee, \& Brody, 1975; Swartzwelder, Richardson, MarkwieseFoerch, Wilson, \& Little, 1998; York \& Chan, 1994) is consistent with both the conditioning analysis of tolerance and results of previous research concerning glucose enhancement of CR formation. The acquisition of classically 'conditioned responses is slower in older than in younger rats and humans (see, e.g., Buchanan \& Ginn, 1988; Kubanis \& Zornetzer, 1981; Prescott, Buchanan, \& Powell, 1989; Weiss \& Thompson, 1991; Zornetzer, Thompson, \& Rogers, 1982). If CRs partially mediate tolerance, it would indeed be expected that the rate of tolerance acquisition to a variety of drugs would be inversely related to the subject's age. Results of research with rats (e.g., Winocour, 1995, 1998) and humans (e.g., Manning 
et al., 1997) suggest that the beneficial effects of glucose on learning are most apparent in older subjects (perhaps because of glucometabolic alterations accompanying aging); thus, we might expect that glycemic modulation of drug tolerance would be especially pronounced in older subjects.

\section{REFERENCES}

Alvarez, R., Zas, R., Fernández-Nova, L., García, M., Polo, E., Détolle-Sarbach, S., GueZ, D., \& CaCabelos, R. (1997). Comparative effects of $\$ 9977-2$ versus tacrine in passive avoidance learning and psychomotor activity. Human Psychopharmacology, 12, 329335.

Arvola, A., Sammalisto, L., \& Wallgren, H. (1958). A test for level of alcohol intoxication in the rat. Quarterly Journal of Studies on Alcohol, 19, 563-572.

Buchanan, S. L., \& GinN, S. R. (1988). Classically conditioned cardiac responses in "old" and "young" Fischer 344 rats. Psychology \& Aging, 3, 51-58.

Cunningham, C. L., Losli, S. M., \& Risinger, F. O. (1992). Contextdrug pairings enhance tolerance to ethanol-induced disruption of operant responding. Psychopharmacology, 109, 217-222.

Dafters, R., Hetherington, M., \& MCCartney, H. (1983). Blocking and sensory preconditioning effects in morphine analgesic tolerance: Support for a Pavlovian conditioning model of drug tolerance. Quarterly Journal of Experimental Psychology, 35B, 1-11.

DEws, P. B. (1962). Psychopharmacology. In A. J. Bachrach (Ed.), Experimental foundations of clinical psychology (pp. 423-441). New York: Basic Books.

DWORKIN, B. R. (1993). Learning and physiological regulation. Chicago: University of Chicago Press.

Epstein, L. H., Caggiula, A. R., Perkins, K. A., McKenzie, S. J., \& SMith, J. A. (1991). Conditioned tolerance to the heart rate effects of smoking. Pharmacology, Biochemistry \& Behavior, 39, 15-19.

FENNESSY, M. R., \& LEE, J. R. (1975). The assessment of and the problems involved in the experimental evaluation of narcotic analgesics. In S. Ehrenpreis \& A. Neidle (Eds.), Methods in narcotics research (pp. 73-99). New York: Marcel Dekker.

Goodison, T., \& SiEgEL, S. (1995). Learning and tolerance to the intake-suppressive effect of cholecystokinin in rats. Behavioral Neuroscience, 109, 62-70.

HAYES, R. L., \& MAYER, D. J. (1978). Morphine tolerance: Is there evidence for a conditioning model? Science, 200, 343-344.

Hinson, R. E., \& SiEgel, S. (1986). Pavlovian inhibitory conditioning and tolerance to pentobarbital-induced hypothermia in rats. Journal of Experimental Psychology: Animal Behavior Processes, 12, 363-370.

KoPF, S. R., \& BARATTI, C. M. (1994). Memory-improving actions of glucose: Involvement of a central cholinergic muscarinic mechanism. Behavioral \& Neural Biology, 62, 237-243.

Krank, M. D., Hinson, R. E., \& SiEgel, S. (1984). The effect of partial reinforcement on tolerance to morphine-induced analgesia and weight loss in the rat. Behavioral Neuroscience, 98, 79-95.

Kubanis, P., \& ZorNetZer, S. F. (1981). Age-related behavioral and neurobiological changes: A review with an emphasis on memory. $B e-$ havioral \& Neural Biology, 31, 115-172.

LARSON, S. J., \& Siegel, S. (1998). External inhibition of ethanolinduced ataxia and compensatory hypertaxia. Pharmacology, Biochemistry \& Behavior, 61, 131-142.

Manning, C. A., Parsons, M. W., Cotter, E. M., \& Gold, P. E. (1997). Glucose effects on declarative and nondeclarative memory in healthy elderly and young adults. Psychobiology, 25, 103-108.

Mansfield, J. G., \& CunNingham, C. L. (1980). Conditioning and extinction of tolerance to the hypothermic effect of ethanol in rats. Journal of Comparative \& Physiological Psychology, 94, 962-969.

Mayfield, R. D., Grant, M., Schallert, T., \& Spirduso, W. W.
(1992). Tolerance to the effects of ethanol on the speed and success of reaction time responding in the rat: Effects of age and intoxicated practice. Psychopharmacology, 107, 78-82.

NICAK, A., \& KoHUT, A. (1978). Development of tolerance to morphine and pethidine in rats in dependence on age. Activitas Nervosa Superior, 20, 231-235.

NozAKI, M., AKera, T., LeE, C. Y., \& Brody, T. M. (1975). The effects of age on the development of tolerance to and physical dependence on morphine in rats. Journal of Pharmacology \& Experimental Therapeutics, 192, 506-512.

OKaICHI, Y., \& OKaICHI, H. (1997). Posttraining glucose in inhibitory avoidance facilitates memory consolidation in rats. Psychobiology, 25, 352-356.

Poulos, C. X., \& CAPPElL, H. (1991). Homeostatic theory of drug tolerance: A general model of physiological adaptation. Psychological Review, 98, 390-408.

Prescott, L., Buchanan, S. L., \& Powell, D. A. (1989). Leg flexion conditioning in the rat: Its advantages and disadvantages as a model system of age-related changes in associative learning. Neurobiology of Aging, 10, 59-65.

RAMSAY, D. S., \& Woods, S. C. (1997). Biological consequences of drug administration: Implications for acute and chronic tolerance. Psychological Review, 104, 170-193.

SIEGEL, S. (1978). Is there evidence for a conditioning model? Science, 200, 344-345.

SiEgel, S. (1983). Classical conditioning, drug tolerance, and drug dependence. In Y. Israel, F. B. Glaser, H. Kalant, R. E. Popham, W. Schmidt, \& R. G. Smart (Eds.), Research advances in alcohol and drug problems (Vol. 7, pp. 207-246). New York: Plenum.

SiEGEL, S. (1991). Feedforward processes in drug tolerance. In R. G. Lister \& H. J. Weingartner (Eds.), Perspectives in cognitive neuroscience (pp. 405-416). New York: Oxford University Press.

Siegel, S., \& Allan, L. G. (1998). Learning and homeostasis: Drug addiction and the McCollough effect. Psychological Bulletin, 124, 230-239.

SIEGEL, S., \& LARSON, S. J. (1996). Disruption of tolerance to the ataxic effect of ethanol by a novel stimulus. Pharmacology, Biochemistry \& Behavior, 55, 125-130.

Swartzwelder, H. S., Richardson, R. C., Markwiese-Foerch, B. WILSON, W. A., \& LITTLE, P. J. (1998). Developmental differences in the acquisition of tolerance to ethanol. Alcohol, 15, 311-314

Tariot, P. N., Patel, S. V., Cox, C., \& Henderson, R. E. (1996). Agerelated decline in central cholinergic function demonstrated with scopolamine. Psychopharmacology, 125, 50-56.

TiffanY, S. T., \& Baker, T. B. (1981). Morphine tolerance in the rat: Congruence with a Pavlovian paradigm. Journal of Comparative \& Physiological Psychology, 95, 747-762.

WALTER, T. A., \& RICCIO, D. C. (1983). Overshadowing effects in the stimulus control of morphine analgesic tolerance. Behavioral Neuroscience, 97, 658-662.

Weiss, C., \& ThOMPSON, R. F. (1991). The effects of age on eyeblink conditioning in the freely moving Fischer-344 rat. Neurobiology of Aging, 12, 249-254.

WINOCOUR, G. (1995). Glucose-enhanced performance by aged rats on a test of conditional discrimination learning. Psychobiology, 23, 270276.

Winocour, G. (1998). Glucose treatment attenuates spatial learning and memory deficits of aged rats on tests of hippocampal function. Neurobiology of Aging, 19, 233-241.

YoRK, J. L., \& ChAN, A. W. K. (1994). Age effects on chronic tolerance to ethanol hypnosis and hypothermia. Pharmacology, Biochemistry \& Behavior, 49, 371-376.

ZORNETZER, S. F., THOMPSON, R., \& Rogers, J. (1982). Rapid forgetting in aged rats. Behavioral \& Neural Biology, 36, 49-60.

(Manuscript received September 1, 1998; revision accepted for publication January 13, 1999.) 